\title{
The Double Chooz experiment
}

\author{
Christian Buck* on behalf of the Double Chooz Collaboration \\ Max-Planck-Institut für Kernphysik Heidelberg, 69117 Heidelberg, Germany \\ E-mail: Christian.Buck@mpi-hd.mpg.de
}

The main goal of the Double Chooz reactor neutrino experiment is a precision measurement of the neutrino mixing angle $\Theta_{13}$. This smallest of the three known mixing angles is determined from the disappearance of electron antineutrinos at a distance of about $1 \mathrm{~km}$ from two nuclear reactors in $\mathrm{Chooz}$, France. Neutrino interactions with the protons of $\mathrm{H}$ atoms in an organic liquid scintillator produce a coincidence signal of a prompt positron and a delayed neutron allowing efficient background suppression. The value of $\Theta_{13}$ is extracted from a background model independent rate only analysis as well as from a fit to the observed energy spectrum. The latter gives a result of $\sin ^{2}\left(2 \Theta_{13}\right)=0.090_{-0.029}^{+0.032}$. In the energy region around $4-6 \mathrm{MeV}$ a spectral distortion compared to the reactor neutrino flux prediction is observed and discussed. This distortion however has no significant impact on the determination of $\Theta_{13}$.

XVI International Workshop on Neutrino Telescopes,

2-6 March 2015

Palazzo Franchetti - Istituto Veneto, Venice, Italy

${ }^{*}$ Speaker. 


\section{Neutrino mixing at nuclear reactors}

Nuclear reactors are a strong, pure and free source of electron antineutrinos which are emitted isotropically. Therefore the vicinity of reactors is a suitable location for neutrino experiments and to study neutrino properties. The history of reactor neutrino experiments already started with the first measurement of a neutrino by Reines and Cowan [1] in the 50ies of the last century.

Since then many experiments followed, most of them placed at distances around $100 \mathrm{~m}$ and below. For a long time these short baseline experiments seemed to be in good agreement with reactor flux predictions and set stringent limits for neutrino mixing at mass splittings of more than $1 \mathrm{eV}$. In 2011, a reevaluation of the reactor flux prediction [2,3] lead to a deficit in the observed neutrino rate, which can not be explained in the standard framework of 3 neutrino families.

Together with solar neutrino experiments the KamLAND reactor neutrino experiment [4] measured the neutrino mixing angle $\Theta_{12}$. For the energy of reactor neutrinos the first oscillation minimum for the "solar parameters" is observed at baselines of about $50 \mathrm{~km}$. In between the short baseline experiments and the KamLAND distance there is a class of experiments with baselines of $1-2 \mathrm{~km}$, which is sensitive to the neutrino mixing angle $\Theta_{13}$.

Whereas the first generation of these experiments, Chooz [5] and Palo Verde [6], could just set upper limits on the smallest of the three mixing angles, a new generation, including Double Chooz [7], Daya Bay [8] and RENO [9], with improved statistical and systematical uncertainties was able to measure $\Theta_{13}$. The key feature of these new experiments was the concept of having near and far detectors. By a comparison of the flux at the near and far detector a pure measurement of $\Theta_{13}$ can be achieved without relying on flux predictions, which typically dominate the uncertainty and sensitivity in such a measurement.

This concept is illustrated for the case of Double Chooz in Figure 1. The near detector at a distance of about $400 \mathrm{~m}$ measures the neutrino rate at a position where the oscillation effect is not yet relevant. The far detector at about $1 \mathrm{~km}$ distance sits close to the first oscillation minimum and detects the disappearance of the electron antineutrinos. There are advantages of such a rather simple site configuration with just two reactors and two detectors almost at iso-flux positions. First the flux related uncertainty is negligible compared to other uncertainties as efficiency and backgrounds. Moreover, the experiment profits from the opportunity to measure the backgrounds in periods with both reactors off [10].

\section{The Double Chooz detector}

The far laboratory of the Double Chooz experiment is located at an average distance of $1.05 \mathrm{~km}$ from the two Chooz reactors. It is the same detector position of the former Chooz experiment with a rock shielding of 300 mw.e. The near detector laboratory was specially built for Double Chooz at an average distance $0.41 \mathrm{~km}$. The rock shielding of the near laboratory is 115 mw.e.

The design of the inner detectors is identical in near and far position. The neutrinos are detected in an inverse beta decay reaction on protons of the hydrogen atoms in an organic liquid target scintillator (Target). The interaction signature of neutrinos with energies above the reaction threshold of $1.8 \mathrm{MeV}$ is a coincidence signal of a prompt positron and a delayed neutron capture. From the measured positron energy deposition in the Target the neutrino energy can be extracted. 


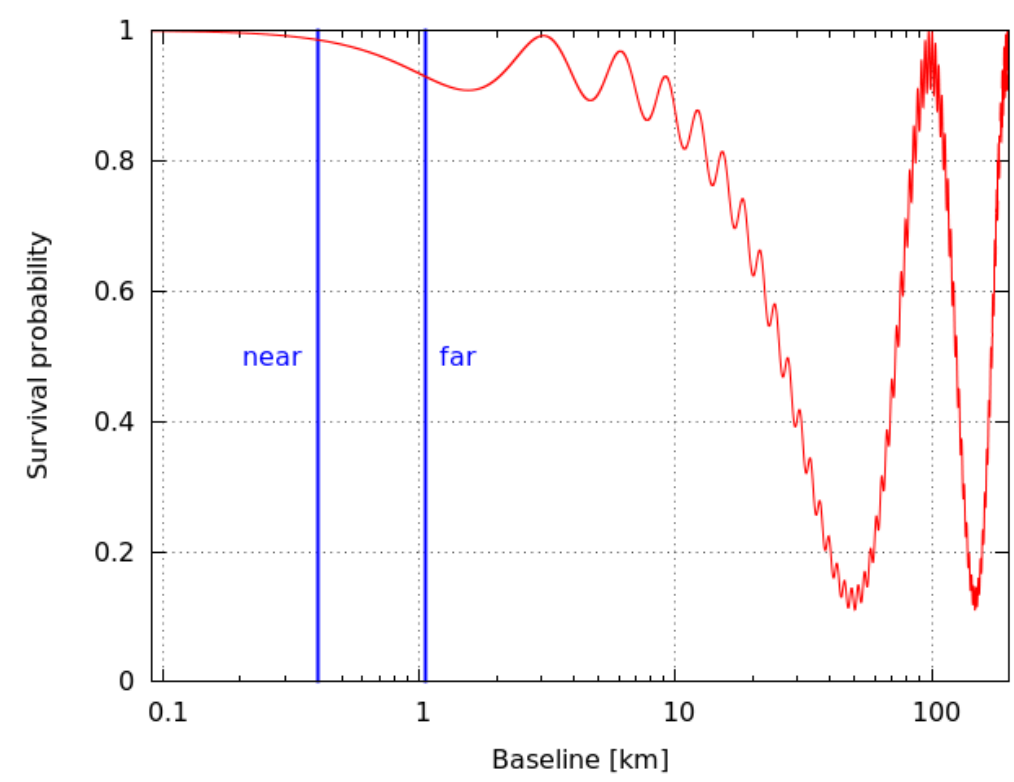

Figure 1: The survival probability of an electron antineutrino is plotted versus the distance from the reactor source position for an energy of $3 \mathrm{MeV}$. The positions of the Double Chooz near and far detector are indicated by the vertical bars.

To keep the coincidence time short (about $30 \mu$ s) and to increase the energy of the gammas emitted in the neutron capture $(8 \mathrm{MeV})$, the target scintillator is doped with $1 \mathrm{~g} / \mathrm{l}$ of gadolinium $(\mathrm{Gd})$.

The Target with a mass of 8.3 tons is contained in an $8 \mathrm{~mm}$ thick cylindrical acrylic vessel and surrounded by about 18 tons of Gd-free liquid scintillator called "Gamma Catcher" (GC). Gammas of the positron annihilation in the Target as well as after the neutron capture on Gd might escape the target volume, but can still be detected in the GC. The liquids of the Inner Detector are completed by 80 tons non-scintillating buffer oil outside the acrylic vessel of the GC. This mineral oil is contained in a steel vessel that optically separates the Inner Detector from a Muon Veto system. At the inner walls of this steel vessel each detector is equipped with 390 photomultiplier tubes (PMTs) of 10 inch.

Two systems are available for muon detection. The so-called "Inner Veto" (IV) consists of 70 tons of linear aklyl benzene (LAB) based liquid scintillator. The scintillation light is observed by 78 PMTs of 8 inch. In addition there are flat layers of plastic scintillator strips on top of the detector, the "Outer Veto" (OV). The shielding strategy is different in the two detectors. The far detector is surrounded by $15 \mathrm{~cm}$ of steel whereas the near detector is shielded against natural radioactivity from the rock by $1 \mathrm{~m}$ of water.

Several systems are available for detector calibration. There are weekly calibrations with a multi wavelengths LED light injection system to monitor the PMT behaviour as gain variations. Radioactive gamma sources, a californium (Cf) neutron source and a laser ball can be deployed inside the Target along the vertical z-axis in the detector center. The radioactive sources can also be positioned at various locations inside the GC routing them along a stainless steel guide tube fixed to the acrylic vessels. 


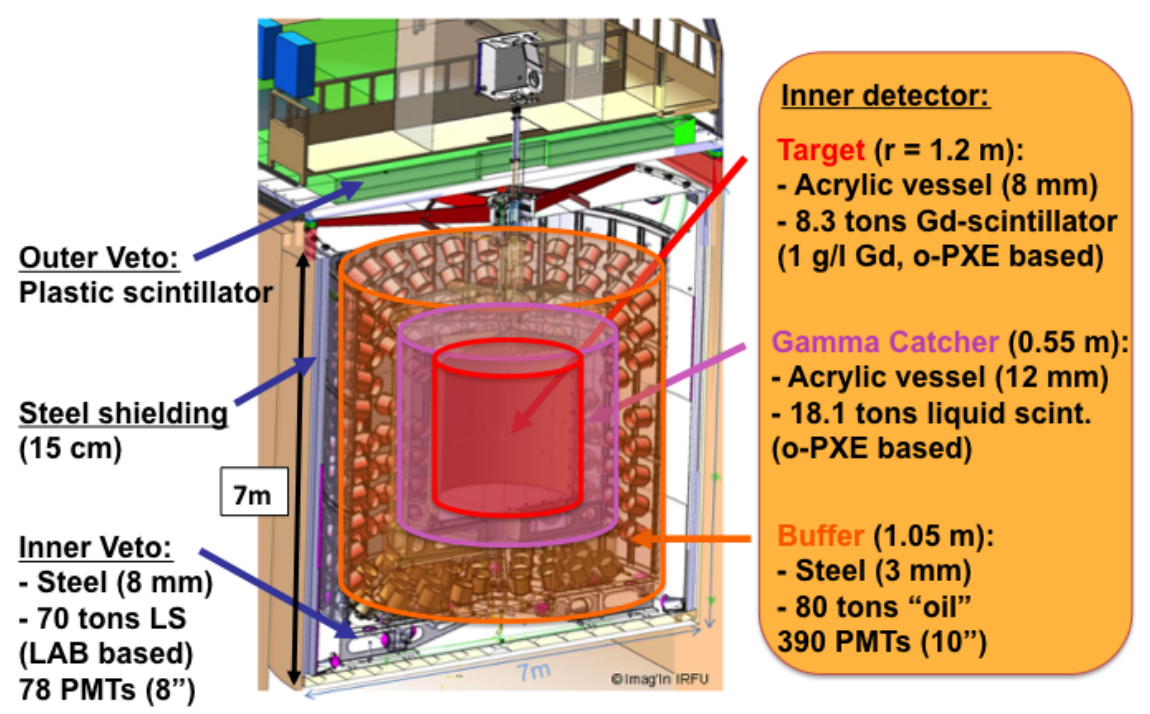

Figure 2: The Double Chooz far detector.

\section{Energy reconstruction}

A precise knowledge of the energy scale is of crucial importance in particular for the $\Theta_{13}$ analysis using the spectral information. The visible energy in the detector is reconstructed from the number of photoelectrons with several corrections. These correction factors take care of nonuniformity of the detector response, non-linearities, time variations and the absolute energy scale.

In the uniformity calibration a correction is applied as a function of the radial and vertical positions to convert the number of photoelectrons for each position to the one at the center of the detector. The calibration is done using the single $2.2 \mathrm{MeV}$ gamma after neutron capture on hydrogen. This correction factor ranges up to $5 \%$ inside the Target. The correction map is applied for the data as well as in the Monte Carlo simulation.

A bias of the baseline estimation can result in gain non-linearities especially at low charges of few photoelectrons. Gain and gain non-linearity of all channels is measured using the LED-fiber calibration system with different light intensities and light injection positions. The gain and its nonlinearity can change after power cycles of the readout electronics, therefore the gain is measured upon each power-cycle period.

Other non-linearities are introduced either by the the modeling of the readout system and charge integration algorithm or by the scintillator liquids. Scintillation light is not linear to the energy deposited in the detector due to two effects. The first is ionization quenching at low electron or positron energies, the second Cerenkov light production. The contribution of the latter to the total light production increases with increasing energies.

The visible energy of the data is corrected for time variations of the mean gain and detector 
response. The observed variations are on the per cent level with some energy dependence of the magnitude. The time variations are extracted and corrected using the neutron capture peak on $\mathrm{H}$ $(2.2 \mathrm{MeV})$. The systematic uncertainty and energy dependence is then studied using data from neutron captures on $\mathrm{Gd}(8 \mathrm{MeV})$ and alphas from ${ }^{212}$ Po-decay $(8.8 \mathrm{MeV})$ which show up around $1 \mathrm{MeV}$ visible energy due to quenching effects.

To determine the absolute energy scale data of the ${ }^{252} \mathrm{Cf}$ source at the detector center is used. From the position of the $2.2 \mathrm{MeV}$ peak of neutrons captured on $\mathrm{H}$ the absolute energy scale is found to be 186 photoelectrons with good agreement between data and Monte Carlo. In total the systematic uncertainty on the energy scale was calculated to be $0.74 \%$ [11].

\section{Neutrino selection}

In the Gd analysis of Double Chooz [11] there is the coincidence signal of the prompt positron event and the high energy ( $8 \mathrm{MeV}$ ) delayed event after neutron capture on $\mathrm{Gd}$, well above the energies of natural radioactivity. For "physics events" we first require that the light signal arrives uniform in space and simultaneous in time at the PMTs. This is to avoid background events due to instrumental light production from the PMT bases.

For the reduction of cosmogenic backgrounds, events within a $1 \mathrm{~ms}$ time window after a muon and prompt events with a coincident signal in the OV are rejected. Compared to previous analyses $[7,12]$ the prompt energy window was enlarged to $0.5-20 \mathrm{MeV}$. Also the delayed energy window and the coincidence time could be widened due to improved background rejection techniques, which in consequence increased the detection efficiency and the signal to noise ratio. The delayed energy window is from $4-10 \mathrm{MeV}$ and the $\Delta \mathrm{t}$ cut from $0.5-150 \mu \mathrm{s}$. No extra triggers were allowed $200 \mu$ s before and $600 \mu$ s after the prompt candidate.

Several new cuts were introduced to reduce backgrounds. The prompt and delayed event must be reconstructed within less than $1 \mathrm{~m}$ distance. Moreover, information obtained by the IV and OV as well as from vertex reconstruction are used to tag and veto correlated background events. With all these selection cuts 17351 neutrino candidates could be observed in 460.7 days of live time. With the modified and optimized selection cuts the signal to background ratio in Double Chooz increased to more than 20 .

\section{Detection efficiency}

The main source of inefficiency in the neutrino detection of Double Chooz is related to the neutron capture. The trigger efficiency reaches $100 \%$ at $500 \mathrm{keV}$ with negligible uncertainty. Also the detection efficiency of the prompt event is close to $100 \%$. The systematic uncertainty associated with the detection of the delayed signal is estimated with ${ }^{252} \mathrm{Cf}$ calibration data and IBD candidate events. The Double Chooz simulation is checked and corrected if needed for the fraction of captures on $\mathrm{Gd}$ and the $\Delta \mathrm{t}, \Delta \mathrm{E}$ selection efficiency based on the results of our data.

The neutrons produced in the IBD reaction are basically captured either on $\mathrm{Gd}$ or on $\mathrm{H}$. There is just a small contribution from captures on $\mathrm{C}$. The fraction of neutron captures on Gd is measured using ${ }^{252} \mathrm{Cf}$ calibration data at the detector center. In the spontaneous fission of the $\mathrm{Cf}$, gammas (prompt signal) as well as neutrons (delayed signal) are emitted. The background in 
this calibration data can be effectively reduced by a prompt cut at $4 \mathrm{MeV}$ or by using a multiplicity condition. In most cases there is more than one neutron emitted per fission. At the Gdconcentration used in Double Chooz the fraction of neutron captures on Gd was determined to be $85.30 \pm 0.08 \%$. To match the result of the simulation to the one of the data a correction factor of $0.9750 \pm 0.0011$ (stat) \pm 0.0041 (syst) was applied.

The neutron selection efficiency over the full detector volume was evaluated using two independent methods. One with calibration data from the neutron source and the other using IBD candidates. Since the data was in very good agreement with the results of the simulations for both methods no correction was applied. The associated uncertainty was determined to be $0.19 \%$ only.

Finally, there is a class of events in which the neutron is detected in a different volume than the positron. For example there can be an IBD reaction in the Gamma Catcher with the positron depositing the energy there, whereas the neutron might travel to the target and can be captured on Gd. When neutrons enter the Target we call it "spill-in" events, for neutrons leaving the Target to the GC we call it "spill-out" events. Since there are more spill-in than spill-out events there is an effective increase of the fiducial volume in the detector beyond the Target. The net spill-in flux is $2.08 \%$. The related systematic uncertainty is determined by the comparison of different simulation models and parameter variations in the MC. As a result the uncertainty was determined to be $0.27 \%$. Combining the three contributions and adding other uncertainties as e.g. the knowledge on the target proton number, we end up with a total uncertainty for the efficiency part of $0.6 \%$, a major improvement compared to previous DC analyses.

\section{Backgrounds}

The backgrounds in Double Chooz can be classified in three categories. One is accidental background which has the smallest contribution of all backgrounds and can be determined with very high precision. Then there are two categories of correlated backgrounds. The first of those are fast neutrons and stopping muons and the second one long-lived cosmogenic isotopes undergoing $\beta$-n-decays. Other contributions, as background due to $(\alpha, n)$ reactions, were found to be negligible and do not impact our $\Theta_{13}$ analysis [11].

\subsection{Accidental background}

As accidental background we name random coincidences of events that meet the conditions of the energy cuts and fall within the coincidence time window. The rate of this type of background is effectively reduced with the distance cut and it can be measured with high precision by shifting the coincidence time window by more than $1 \mathrm{~s}$ from the prompt candidate. In this way the background rate is measured to be $0.0701 \pm 0.0003$ (stat) \pm 0.0026 (syst) events/day. This rate is found to be stable over the data taking period.

\subsection{Fast neutrons and stopping muons}

Correlated signals of fast neutrons (FN) or stopping muons (SM) are another source of background in DC. Fast neutrons can be produced by muons missing the veto systems of the detector and producing spallation neutrons. The neutrino signal can then be mimicked by these neutrons entering the detector from outside, creating recoil protons from neutron scattering (prompt event). 
The neutron itself (or another one) can then be captured in the fiducial volume of the detector and create the delayed signal. The SM events arise from muons stopping and decaying in the detector without being tagged by the veto systems. Typically these muons enter the detector via the chimney. Here the muon track mimics the prompt event and the decay Michel electron the delayed.

Background from SM can be largely suppressed using information from the vertex reconstruction, which assumes point like events in the central part of the detector. Those differ in PMT time and charge distribution from muons or high energy electron events in the chimney area. Therefore the background from FN is significantly higher. The spectral shape of the FN background is checked using events, which are tagged by the IV, since the neutrons often deposit some energy in the IV scintillator before entering the inner detector. From these IV tagged events and a high energy fit from the upper end of the neutrino spectrum (around $12 \mathrm{MeV}$ ) to $30 \mathrm{MeV}$, a region where correlated background dominates, a shape consistent with a flat spectrum was found and no evidence for any energy dependence observed. The flat shape is also confirmed by events vetoed by the OV. The correlated background rate is $0.604 \pm 0.051$ events/day estimated by extrapolation from coincidences between 20 and $30 \mathrm{MeV}$.

\subsection{Cosmogenic isotopes}

The highest background in DC with largest uncertainty is coming from cosmogenically produced $\beta$-n emitters like the dominant ${ }^{9} \mathrm{Li}$ or ${ }^{8} \mathrm{He}$. Since the half life of these isotopes is above $100 \mathrm{~ms}$, it is impossible to veto them without introducing significant dead-time. The contribution of this background is evaluated by fitting the time correlation between the IBD candidates and the previous muons. The probability to produce ${ }^{9} \mathrm{Li}$ increases with the muon energy. Therefore the most precise fit result is obtained using muons depositing more than $600 \mathrm{MeV}$ in the detector. At lower energies an additional cut on the distance of the muon tracks to the prompt vertex is applied to reduce accidental pairs.

A lower limit for this background was computed separately by selecting a Li-enriched sample taking into account muon energy information, lateral distances to the muon track and production of additional neutrons in the muon interactions. In this way the uncertainty to the lower side could be significantly reduced and an assymetric uncertainty is obtained. After subtracting Li events rejected by a dedicated veto, the final background rate from cosmogenic isotopes is $0.97_{-0.16}^{+0.41}$ events/day. The spectral shape was determined from Li candidate events including those with neutrons from the IBD captured on $\mathrm{H}$ to improve statistics. The spectrum is consistent with the one obtained from simulation studies.

\subsection{Reactor off-off measurements}

One of the unique features in the Double Chooz experiment is the possibility of measuring the background in reactor off periods. So far there were two of these reactor off periods during the far detector data taking. In total 57 neutrino candidates were measured during 7.24 days of live-time. After applying all the vetoes 7 events remained. From dedicated simulation studies $1.57 \pm 0.47$ events are expected from residual reactor neutrinos. From the sum of residual neutrinos and background expectations a slightly higher rate of $12.9_{-1.4}^{+3.1}$ events is predicted in the off-off period. The compatibility to the observed number of events is $9.0 \%(1.7 \sigma)$. This reactor off-off result is used to constrain the total background rate in the neutrino oscillation analyses. 


\section{Neutrino oscillation analysis}

In the following the results of two $\Theta_{13}$ analyses [11] are presented. The first includes energy information from the shape of the positron spectrum, whereas the second is a dedicated rate only analysis. Both methods give consistent results demonstrating the robustness of the $\Theta_{13}$-analysis in Double Chooz. Higher precision is obtained from the rate + shape analysis, which is discussed first.

\subsection{Rate + shape analysis}

This oscillation analysis is based on a fit to the antineutrino rate and spectral shape. Data are compared to the prediction of an antineutrino MC sample. The data are separated into 40 energy bins between 0.5 and $20 \mathrm{MeV}$. The prediction for the number of events including neutrino signal and background is constructed for each bin. Systematic and statistical uncertainties are propagated to the fit by the use of a covariance matrix in order to properly account for correlations between energy bins. From the fit information it is possible to further constrain the background, in particular due to the data points above $8 \mathrm{MeV}$, where the number of predicted neutrino events is very small.

The analysis considers the uncertainties on $\Delta \mathrm{m}^{2}$, the number of residual neutrinos in the reactor-off periods, the backgrounds and the energy scale represented by three parameters. A scan of $\chi^{2}$ is carried out over a wide range of $\sin ^{2}\left(2 \Theta_{13}\right)$, minimizing it with respect to eight fit parameters for each value of the mixing angle. The minimum $\chi^{2}$ value is found at $\sin ^{2}\left(2 \Theta_{13}\right)=0.090_{-0.029}^{+0.032}$ $\left(\chi^{2} /\right.$ d.o.f. $\left.=52.2 / 40\right)$. Several cross-checks were carried out, e.g. removing the constraint to fit parameters for the correlated background rates or a rate only fit. Consistent values of $\Theta_{13}$ were obtained indicating the robustness of this fit.

\subsection{Reactor rate modulation analysis}

The mixing angle $\Theta_{13}$ is also determined in Double Chooz using a special rate only analysis. In this case, observed and expected neutrino candidate rates are compared for different reactor power conditions. There are mainly three different configurations: both reactors on, one reactor off or both reactors off. Furthermore, the reactors are not always running at full power. The observed candidate rate is plotted versus the expected rate in seven bins for different reactor power conditions. From a linear fit the mixing angle as well as the background rate can be estimated. This analysis can be performed either independent of any background model using just the reactor off-off measurement or constraining the background using the estimated rates as described in the Backgrounds section.

Highest sensitivity on $\Theta_{13}$ is obtained including the constraint on the total background rate of $1.64_{-0.17}^{+0.41}$ events/day. The best-fit value for $\sin ^{2}\left(2 \Theta_{13}\right)$ is found to be $0.090_{-0.035}^{+0.034}$ in perfect agreement to the rate + shape fit result. If the constraint on the background rate is removed and the analysis is done just using the background information from the measurement with about seven days of both reactors off, a slightly lower value of the mixing angle is obtained. In this cross check analysis we get $\sin ^{2}\left(2 \Theta_{13}=0.060 \pm 0.039\right.$ and as additional fit output a lower rate on the total background rate of $0.93_{-0.36}^{+0.43}$ events/day. 


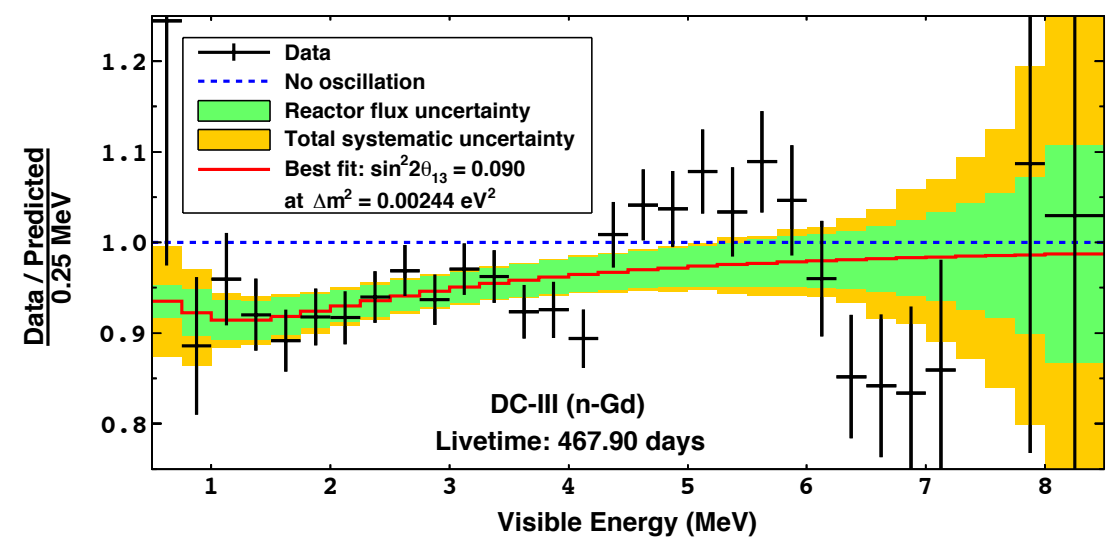

Figure 3: Black points show the ratio of the data to the prediction without oscillations. The red line shows the best-fit including $\Theta_{13}$ with the reactor flux uncertainty (green band) and the total systematic uncertainty (orange band).

\section{Spectral distortion}

A spectral distortion compared to the predicted reactor flux besides the oscillatory signature is found above $4 \mathrm{MeV}$ of the prompt energy as shown in Figure 3 [11]. There is an excess of the observed rate around $5 \mathrm{MeV}$. The significance of this excess was evaluated to be on the $3 \sigma$ level. The impact of the distortion on the $\Theta_{13}$ analysis of Double Chooz was studied and found to be small. For the oscillation analysis in Double Chooz the energy region below $4 \mathrm{MeV}$ is much more relevant, since the oscillation effect is expected mainly at low energies.

The energy scale in this region is confirmed by spallation neutrons captured on carbon (energy peak at $5 \mathrm{MeV}$ ), for which data and MC simulation agree within $0.5 \%$. Furthermore, no distortion is observed in the comparison of the ${ }^{12} \mathrm{~B}$ energy spectrum of data and MC simulation. Those observations disfavor the energy scale as cause of the distortion. The rate of the excess is found to be strongly correlated to the reactor power, which is not expected in case it is caused by a so far unknown background source in the $5 \mathrm{MeV}$ region. Therefore, the hypothesis of an additional background contribution in this region is disfavored as well. There are investigations, if the excess is caused by limitations of the reactor flux predictions, but those studies are not yet conclusive.

The excess in the $5 \mathrm{MeV}$ region is also seen in other $\Theta_{13}$ experiments as RENO and Daya Bay. This is an additional hint that the cause is probably not background or detector related, since the systematics of the experiments is rather different.

\section{Prospects with near detector}

Double Chooz has finalized its near detector (ND) construction by the end of 2014 and first preliminary data demonstrated the feasibility of the reactor neutrino measurement with both detectors. So far, with one detector only, the dominant uncertainty in Double Chooz is on the reactor flux prediction. This will change completely with the ND, since the flux uncertainty is then strongly 
suppressed due to the simple experimental set up of the Double Chooz site with only two reactors and the detectors positioned almost on the iso-flux line. Based on the current knowledge the projected sensitivity with the ND reaches $\sigma\left(\sin ^{2}\left(2 \Theta_{13}\right)\right)=0.015$ after few years and could reach 0.010 with further analysis improvements.

\section{Conclusion}

The Double Chooz reactor neutrino experiment has improved its analysis on the neutrino mixing angle $\Theta_{13}$ with the far detector only. The accuracy of the energy reconstruction could be improved significantly, the neutrino selection was optimized to reduce systematics, new background tagging and rejection techniques were implemented and data with both reactors off were included. As a result a value for $\sin ^{2}\left(2 \Theta_{13}\right)$ of $0.090_{-0.029}^{+0.032}$ is found in an analysis using rate and shape information. The result was confirmed in a spectrum and background independent rate only analysis comparing the observed and expected neutrino rate for different reactor power conditions. A distortion of the energy spectrum compared to predictions beyond the oscillation effect was observed. Data taking with the near detector has started and further significant improvements of the Double Chooz sensitivity are expected.

\section{References}

[1] F. Reines and C.L. Cowan, Detection of the Free Neutrino, Phys.Rev. 92, 930-931 (1953).

[2] Th.A. Mueller et al., Improved predictions of reactor antineutrino spectra, Phys.Rev. C 83, 054615 (2011).

[3] G. Mention et al., Reactor antineutrino anomaly, Phys.Rev. D 83, 073006 (2011).

[4] T. Araki et al., Measurement of Neutrino Oscillation with KamLAND: Evidence of Spectral Distortion, Phys.Rev.Lett. 94, 081801 (2005).

[5] M. Appolonio et al., Search for neutrino oscillations on a long base-line at the CHOOZ nuclear power station, Eur.Phys.J. C 27, 331-374 (2003).

[6] F. Boehm et al., Final results from the Palo Verde neutrino oscillation experiment, Phys.Rev. D 64, 112001 (2001).

[7] Y. Abe et al., Indication of Reactor $\bar{v}_{e}$ Disappearance in the Double Chooz Experiment, Phys.Rev.Lett. 108, 131801 (2012).

[8] F.P. An et al., Spectral Measurement of Electron Antineutrino Oscillation Amplitude and Frequency at Daya Bay, Phys.Rev.Lett. 112, 061801 (2014).

[9] J.K. Ahn et al., Observation of Reactor Electron Antineutrinos Disappearance in the RENO Experiment, Phys.Rev.Lett. 108, 191802 (2012).

[10] Y. Abe et al., Direct measurement of backgrounds using reactor-off data in Double Chooz, Phys.Rev. D 87, 011102(R) (2013).

[11] Y. Abe et al., Improved measurements of the neutrino mixing angle $\Theta_{13}$ with the Double Chooz detector, JHEP 10 (2014) 086 [Erratum ibid. 02 (2015) 074].

[12] Y. Abe et al., Reactor $\bar{v}_{e}$ disappearance in the Double Chooz experiment, Phys.Rev. D 86, 052008 (2012). 